\title{
A consequence of the gravitational self-force for circular orbits of the Schwarzschild geometry
}

\author{
Steven Detweiler \\ Institute for Fundamental Theory, Department of Physics, \\ University of Florida, Gainesville, FL 32611-8440*
}

(Dated: April 22, 2008)

\begin{abstract}
A small mass $\mu$ in orbit about a much more massive black hole $m$ moves along a world line that deviates from a geodesic of the black hole geometry by $O(\mu / m)$. This deviation is said to be caused by the gravitational self-force of the metric perturbation $h_{a b}$ from $\mu$. For circular orbits about a non-rotating black hole we numerically calculate the $O(\mu / m)$ effects upon the orbital frequency and upon the rate of passage of proper time on the worldline. These two effects are independent of the choice of gauge for $h_{a b}$ and are observable in principle. For distant orbits, our numerical results agree with a post-Newtonian analysis including terms of order $(v / c)^{6}$.

PACS numbers: $04.25 \mathrm{Nx}, 04.30 .-\mathrm{w}, 04.80 . \mathrm{Nn}, 97.60 . \mathrm{Jd}$
\end{abstract}

\section{INTRODUCTION AND SUMMARY}

\section{A. Gravitational waveforms, perturbation analysis and the self-force}

The push towards the detection of gravitational waves depends upon accurate theoretical models of the sources. Numerical relativity has made great progress in the past few years and now tracks black hole binary systems for many orbits and down to the final coalescence and ring down. These recent computational successes are joining the post-Newtonian analyses in providing building blocks for the construction of gravitational wave templates.

A gap in the theoretical progress remains for binary systems with an extreme mass ratio. A small black hole in a close orbit about a much larger one might be too relativistic for post-Newtonian analyses. And the great difference in length scales for the two black holes causes difficulty for a full numerical relativity solution. This gap is the natural domain of perturbation theory.

The state of the art in perturbative gravitational waveforms [1] has a small mass $\mu$ moving along a geodesic of the metric $g_{a b}$ of the much larger black hole. The perturbed Einstein equations then determine the metric perturbation $h_{a b}$ which contains the waveform. But, these waveforms would be much improved if the influence of $h_{a b}$ back on the motion of $\mu$ were part of the analysis. Then the dissipative effects of radiation reaction, joining with conservative effects, would be reflected in the waveform and would allow tracking $\mu$ for more orbits without losing phase information as the inspiral proceeds.

The metric perturbation $h_{a b}$ causes the worldline of $\mu$ to be a geodesic of $g_{a b}+h_{a b}$. Sometimes the same motion is described as non-geodesic in $g_{a b}$ with the acceleration $a_{\mathrm{SF}}^{a}$ being caused by the self-force from $h_{a b}$ acting on $\mu$. In this paper, we prefer the "geodesic in $g_{a b}+h_{a b}$ "

*Electronic address: det@ufl.edu description of the motion, and use the phrase "self-force" only in a generic way to describe any and all of the $O(\mu)$ effects of $h_{a b}$ on the world-line of $\mu$, even those which are unrelated to the acceleration $a_{\mathrm{SF}}^{a}$.

When the mass $\mu$ is modeled as a point particle, an attempt to calculate any consequence of the self-force immediately bumps against a technical difficulty. The metric perturbation $h_{a b}$ is singular at the location of $\mu$, precisely where it must be evaluated. Some method of regularization must be invoked to remove the singular feature. The formal aspects of this regularization are now very well understood [2-13], and we give some details for our particular application in Sec. IV.

\section{B. Our limitations}

In this paper we limit our analysis to the self-force effects upon the circular orbits of the Schwarzschild geometry. The numerical analysis is straightforward, and the theoretical interpretation of the results is clear and unambiguous. These simple orbits provide a convenient test bed for ideas regarding the self-force.

Despite the emphasis of our opening paragraphs, this paper contains no new information regarding the dissipative effects of radiation reaction on the gravitational waveform for slow, circular inspiral into a non-rotating black hole. In Appendix D we sketch a proof, based upon the form of the perturbed Einstein equation, that the self-force removes energy directly from the orbit of $\mu$, via Eq. (19), at a rate that matches the energy being radiated away in the wave zone and down through the event horizon. This result is satisfying but not unexpected. At this order of approximation, the dissipative effects of the self-force imply that the orbital frequency $\Omega$ should change at a rate

$$
\frac{d \Omega}{d t}=\mu \frac{d E}{d t} \times \frac{d \Omega / d r}{\mu d E / d r}+O\left(\mu^{2}\right)
$$

where $d E / d t$ is evaluated from Eq. (19), and $\mu E$ is the $O(\mu)$ contribution to the mass-energy of the system as 
measured at a great distance, which is equivalent to $\mu T^{a} u_{a}+O\left(\mu^{2}\right)^{1}$, with $T^{a}$ being the timelike Killing vector and $u^{a}$ being the four velocity of $\mu$. Thus, for circular orbits of the Schwarzschild geometry the self-force formalism based upon first-order perturbation theory adds formality but yields nothing new about the evolution of the gravitational waveforms from the dissipative part of the self-force. In fact, conservative self-force effects on the values of $E$ and $\Omega$ for circular orbits are described in Sec. II B but these are not sufficient for improving the calculation of $d \Omega / d t$ from dissipation without at least also determining the $O\left(\mu^{2}\right)$ part of $d E / d t$. This result is common knowledge within the self-force community [16] but appears not to have spread much beyond that.

Non-circular orbits in the Schwarzschild metric and all orbits in the Kerr metric contain substantial complications that we also do not address.

\section{Our results}

In perturbative matters, we prefer to focus upon those observable quantities which can be defined in a manner independent of the gauge choice for $h_{a b}$. The angular velocity $\Omega$ and the time component of the four velocity $u^{t}$ are two such quantities for $\mu$ in a circular orbit. Our main computational result is determining the change in the relationship between $\Omega$ and $u^{t}$ caused by the selfforce. The coordinate radius of the orbit is not included in this analysis because it depends upon the gauge choice and has no inherent physical meaning.

First, we define $R_{\Omega}$ via $\Omega^{2}=m / R_{\Omega}^{3}$ as a natural radial measure of the orbit which inherits the property of gauge independence from $\Omega$. In Sec. III we show that $u^{t}$ naturally separates into two parts $u^{t}={ }_{0} u^{t}+{ }_{1} u^{t}$, where each part is individually gauge independent. Further the functional relationships between $\Omega,{ }_{0} u^{t}$ and $R_{\Omega}$ are identical to their relationships in the geodesic limit, $\mu \rightarrow 0$, and show no effect from the self-force.

The remainder ${ }_{1} u^{t}$ is, however, a true consequence of the self-force, and we plot ${ }_{1} u^{t}$ as a function of $R_{\Omega}$ in Figs. 1 and 2. These two figures along with Table I are the primary numerical results of this paper.

In Appendix E we derive a post-Newtonian (PN) expansion for ${ }_{1} u^{t}$ based upon the work of others $[17,18]$. Our expansion is in powers of $m / R_{\Omega}$, which is $v^{2} / c^{2}$ in the Newtonian limit, and from Eq. (E26)

$$
{ }_{1} u^{t}=\frac{\mu}{m}\left[-\left(\frac{m}{R_{\Omega}}\right)-2\left(\frac{m}{R_{\Omega}}\right)^{2}-5\left(\frac{m}{R_{\Omega}}\right)^{3}+\cdots\right] \text {, }
$$

which includes terms of order $v^{6} / c^{6}$.

\footnotetext{
1 This equivalency follows from Zerilli's [14, 15] analysis of monopole perturbations of the Schwarzschild metric.
}

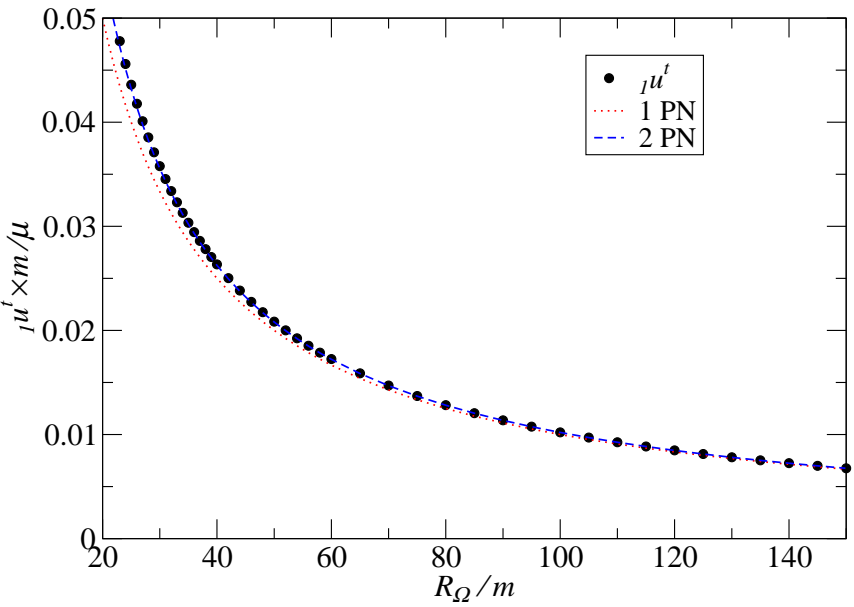

FIG. 1: The quantity ${ }_{1} u^{t}$, which is the gauge independent $O(\mu)$ part of $u^{t}$ effected by the self-force, is given as a function of $R_{\Omega}$ for circular orbits in the Schwarzschild geometry. Also shown are ${ }_{1} u^{t}$ as calculated with $1 \mathrm{PN}$ and $2 \mathrm{PN}$ analyses of Appendix E based upon Refs. [17] and [18].

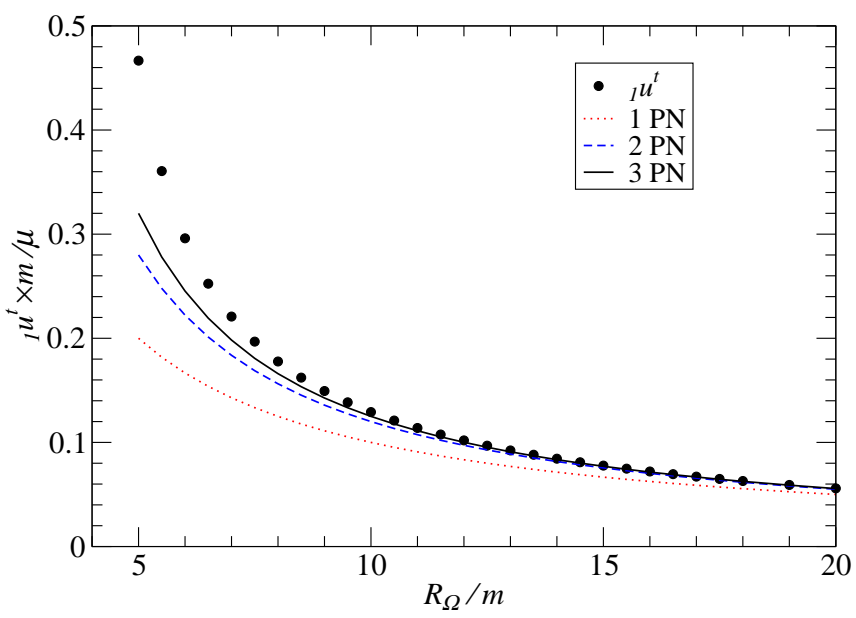

FIG. 2: The same as Fig. 1, but including the 3PN analysis.

Our Figs. 1 and 2 display the different levels of the post-Newtonian expansion. These agree with our numerical results as well as they should.

With a good bit of hubris, we have gone one step further. After the three known post-Newtonian terms were removed from our determined values of ${ }_{1} u^{t}$, we numerically fit the residual to an expansion of higher order terms, and found the coefficient of $\left(m / R_{\Omega}\right)^{4}$ to be $-27.61 \pm .03^{2}$.

We now describe an astronomical thought-observation.

${ }^{2}$ We had already used a similar technique while only knowing the analytic expansion up to the $\left(m / R_{\Omega}\right)^{2}$ term. Finding the coefficient of $\left(m / R_{\Omega}\right)^{3}$ to be -5.001 , with the last digit uncertain, provided the impetus to push the analytical work to one more order. 
Imagine that an observed system has a small mass in a circular orbit, with orbital frequency $\Omega$, about a large non-rotating black hole. Assume that the mass $m$ of the black hole could be determined by some independent means, and that a redshift observation, perhaps as described in Sec. II C 2, could determine $u^{t}$. Then

$$
{ }_{0} u^{t}=\left[1-3(\Omega m)^{2 / 3}\right]^{-1 / 2}
$$

from Eq. (42),

$$
R_{\Omega}=\left(\frac{m}{\Omega^{2}}\right)^{1 / 3}
$$

from the definition of $R_{\Omega}$, and

$$
{ }_{1} u^{t}=u^{t}-{ }_{0} u^{t} .
$$

With values of $m, R_{\Omega}$ and ${ }_{1} u^{t}$ in hand, an astronomer could then determine the mass of the small object from the graphical data.

Our self-force result may only be of academic interest. But it is a result. Our numerical calculations are consistent with a post-Newtonian expansion. We estimate the unknown coefficient of $v^{8} / c^{8}$ in the expansion. And we have proposed one unlikely observational method to infer the mass of a small object which is orbiting a much larger black hole.

\section{Outline}

In Sec. II we introduce our notation, and we provide the components of the geodesic equation for the perturbed Schwarzschild metric $g_{a b}+h_{a b}$ in Sec. II A.

In Sec. II B we focus on quasi-circular orbits - those that would be circular except for the dissipative effects of the self-force. And we find that two components of the four-velocity, $u^{t}$ and $u^{\phi}$, are effected by the self-force in a manner that is $O(\mu)$ and independent of the particular gauge that is chosen for $h_{a b}$. Their ratio is the orbital frequency $\Omega=u^{\phi} / u^{t}$, which is also gauge independent.

The component $u^{t}$ is the ratio of the rate of passage of Schwarzschild time to proper time at the particle. Two different physical interpretations of $u^{t}$ for quasi-circular orbits are given in Sec. II C; one of these is in terms of a redshift measurement, the other is related to the helical Killing vector field of the perturbed geometry.

The formal effect of the self-force on the relationship between $\Omega$ and $u^{t}$ is described in Sec. III. Particular emphasis is placed upon how the gauge independence of this effect reveals itself.

Section IV contains details of our numerical determination of the relationship between $u^{t}$ and $\Omega$. Table I contains an extensive summary of our numerical results which might be used by others for a numerical comparison. This section also has a description of the regularization required by our application which points out some difficulties inherent in regularizing any quantity which is not gauge independent, such as the acceleration of $\mu$ as measured in the background metric.

Our conclusion in Sec. V describes both the current state of self-force calculations as well as its possible future.

We have relegated a number of side discussions to Appendices.

Some convenient identities are given in Appendix A.

Gauge transformations that lead to the understanding that $u^{t}, u^{\phi}$ and $\Omega$ are gauge independent are given in Appendix B.

An elementary, completely Newtonian example of the confusion that gauge freedom can cause is in Appendix C.

In Appendix D we show that the rate at which $\mu$ loses energy via the self-force precisely matches the rate that gravitational waves carry energy out at infinity and down the black hole.

The post-Newtonian expansion for ${ }_{1} u^{t}$ in Eq. (2) is derived in Appendix E.

\section{GEODESICS OF THE PERTURBED SCHWARZSCHILD GEOMETRY}

Self-force analysis implies that a point mass $\mu$ moves along a geodesic of the perturbed Schwarzschild metric $g_{a b}+h_{a b}$ where, in this section, $h_{a b}$ is the suitably regularized metric perturbation and is at least $\mathcal{C}^{1}$. We assume that the geodesic is in the equatorial plane. We assume that $h_{a b}=O(\mu)$, and we work only through first order in $\mu$ or $h_{a b}$. Indices are lowered and raised with $g_{a b}+h_{a b}$ and $g^{a b}-h^{a b}$. We use Schwarzschild coordinates, and $h_{a b}$ is a function of $t, r, \theta$ and $\phi$. To be clear: we specifically choose to normalize the four velocity $u^{a}$ of the particle with respect to the actual regularized physical metric $g_{a b}+h_{a b}$, and not with respect to the abstract background Schwarzschild metric $g_{a b}$.

The quantities $E, \dot{R}$, and $J$ are functions of the proper time $s$ and are defined in terms of the particle's fourvelocity by

$$
E \equiv-u_{t}, \quad \dot{R} \equiv u^{r} \quad \text { and } \quad J \equiv u_{\phi},
$$

which leads to

$$
u_{a}=\left(-E, \frac{\dot{R}+u^{b} h_{b}{ }^{r}}{1-2 m / r}, 0, J\right)
$$

and

$$
u^{a}=\left(\frac{E+u^{b} h_{b t}}{1-2 m / r}, \dot{R}, 0, \frac{J-u^{b} h_{b \phi}}{r^{2}}\right)
$$

where the overdot represents $d / d s$. Thus, $u^{a}$ depends upon $s$ implicitly through its dependence upon $E, \dot{R}, J$ and also upon $r$, evaluated at the particle. These expressions for $u_{a}$ and $u^{a}$ are recursive, but the recursion is intended to be carried only through first order in $h_{a b}$. 
It is convenient to define the non-radial part of the four-velocity of the particle, with no $h_{a b}$ terms included, as

$$
\bar{u}^{a} \equiv\left(\frac{E}{1-2 m / r}, 0,0, \frac{J}{r^{2}}\right) .
$$

Also, the instantaneous angular velocity of the particle is

$$
\Omega \equiv u^{\phi} / u^{t} .
$$

\section{A. Geodesic equation}

One convenient exact form of the geodesic equation for the metric $g_{a b}+h_{a b}$ is

$$
\frac{d u_{a}}{d s}=\frac{1}{2} u^{b} u^{c} \frac{\partial}{\partial x^{a}}\left(g_{b c}+h_{b c}\right) .
$$

While analyzing this geodesic equation we drop all terms of $O\left(\mu^{2}\right)$. The components of Eq. (11) provide

$$
\begin{aligned}
\frac{d E}{d s} & =-\frac{1}{2} u^{a} u^{b} \frac{\partial h_{a b}}{\partial t}, \\
\frac{d J}{d s} & =\frac{1}{2} u^{a} u^{b} \frac{\partial h_{a b}}{\partial \phi} \\
\frac{d}{d s}\left(\frac{r \dot{R}}{r-2 m}+u^{a} h_{a r}\right) & =\frac{1}{2} u^{a} u^{b} \frac{\partial}{\partial r}\left(g_{a b}+h_{a b}\right) .
\end{aligned}
$$

These and other equations describing the particle's worldline are to be evaluated at the location of the particle.

The normalization of $u^{a}$ is a first integral of the geodesic equation,

$$
\begin{aligned}
1 & =-u^{a} u^{b}\left(g_{a b}+h_{a b}\right) \\
& =\frac{E^{2}-\dot{R}^{2}}{1-2 m / r}-\frac{J^{2}}{r^{2}}+\bar{u}^{a} \bar{u}^{b} h_{a b}-\dot{R}^{2} h_{r r}
\end{aligned}
$$

which is derived by beginning with Eq. (8).

\section{The radial component}

Further analysis of the radial component of the geodesic equation is lengthy and requires care. We begin with Eq. (14), and use Eqs. (7) and (8) extensively along with the elementary identities in Appendix A which allow for the easy commutation of $\bar{u}^{a}$ with $\partial_{r}$. Simplification proceeds by identifying all terms which are $O\left(\mu^{2}\right)$ if the motion is quasi-circular as is described in Sec. II B; these terms are systematically moved to the left hand side. Toward the end of the analysis the normalization condition (15) is used to replace $E^{2}$ in favor of $J^{2}$ on the right hand side. Final simplification arises from a convenient placement of the indices of $h_{a b}$ on the left hand side. The result is

$$
\begin{aligned}
\ddot{R}\left(1+h_{r}{ }^{r}\right)+ & \frac{1}{2} \dot{R}^{2} \partial_{r} h_{r}{ }^{r}+\bar{u}^{a} u^{b} \partial_{a} h_{b}{ }^{r}+\dot{E} h_{t r}+\dot{J} h^{\phi r} \\
= & -\frac{m}{r^{2}}\left(1-\bar{u}^{a} \bar{u}^{b} h_{a b}\right)+\frac{J^{2}}{r^{4}}(r-3 m) \\
& +(1-2 m / r) \frac{1}{2} \partial_{r}\left(\bar{u}^{a} \bar{u}^{b} h_{a b}\right) .
\end{aligned}
$$

\section{B. Quasi-circular orbits}

For any orbit Eqs. (12) and (13) imply that $\dot{E} \sim \dot{J} \sim$ $O(\mu)$. In this section our interest focuses on the orbits which would be circular but for these effects of radiation reaction. For these quasi-circular orbits we require that $\dot{R}=O(\mu)$ and also that $\ddot{R}=O\left(\mu^{2}\right)$. The second of these conditions is necessary to insure that the orbit is not a precessing elliptical orbit with a very small eccentricity $\epsilon=O(\mu)$. To summarize, for a quasi-circular orbit $E, R$ and $J$ change slowly, $\dot{E} \sim \dot{R} \sim \dot{J} \sim O(\mu)$, and their rates of change are slower still, $\ddot{E} \sim \ddot{R} \sim \ddot{J} \sim O\left(\mu^{2}\right)$.

In this section we continue to drop $O\left(\mu^{2}\right)$ terms including those which are consequences of the quasi-circular condition from the geodesic equations. Thus the normalization of $u^{a}$ becomes

$$
1=\frac{E^{2}}{1-2 m / r}-\frac{J^{2}}{r^{2}}+\bar{u}^{a} \bar{u}^{b} h_{a b} .
$$

Neither $T^{a} \partial / \partial x^{a}=\partial / \partial t$ nor $\Phi^{a} \partial / \partial x^{a}=\partial / \partial \phi$ is a Killing vector of $g_{a b}+h_{a b}$, but a helical Killing vector $k^{a}$ exists for a quasi-circular orbit in that $\mathcal{L}_{k}\left(g_{a b}+h_{a b}\right)=$ $O\left(\mu^{2}\right)$. The Killing vector exists for any gauge choice for $h_{a b}$. But if the gauge choice is reasonable and $h_{a b}$ respects the helical symmetry, then $k^{a}$ is particularly simple [13] with

$$
\begin{aligned}
k^{a} \partial / \partial x^{a} & =\partial / \partial t+\Omega \partial / \partial \phi, \\
\mathcal{L}_{k} g_{a b} & =0, \text { and } \mathcal{L}_{k} h_{a b}=O\left(\mu^{2}\right) .
\end{aligned}
$$

In what follows, the only assumption which we make about the gauge is that it respects both the helical symmetry over a dynamical timescale and also the natural reflection symmetry through the equatorial plane. Our results are independent of the assumption, but the analysis is much simplified because of it. For example, $\bar{u}^{a}$ is tangent to a trajectory of $k^{a}$ so that $\bar{u}^{a} \partial_{a} h_{b c}=O\left(\mu^{2}\right)$ at the particle for a quasi-circular orbit.

For quasi-circular orbits Eqs. (12) and (13) simply imply that

$$
\begin{aligned}
& \frac{d E}{d t}=-\frac{1}{2 \bar{u}^{t}} \bar{u}^{a} \bar{u}^{b} \partial_{t} h_{a b} \quad \text { and } \\
& \frac{d J}{d t}=\frac{1}{2 \bar{u}^{t}} \bar{u}^{a} \bar{u}^{b} \partial_{\phi} h_{a b},
\end{aligned}
$$

and Eq. (18) then yields

$$
\frac{d E}{d t}=\Omega \frac{d J}{d t} .
$$


We show in Appendix B that the right hand sides of Eqs. (19) and (20) are both independent of the gauge choice for $h_{a b}$. Thus, $d E / d t$ and $d J / d t$ are gauge independent for quasi-circular orbits.

For a quasi-circular orbit every term on the left hand side of the radial equation (16) is $O\left(\mu^{2}\right)$. It easily follows that

$$
\frac{J^{2}}{r^{2}}=\frac{m}{r-3 m}\left(1-\bar{u}^{a} \bar{u}^{b} h_{a b}\right)-\left(\frac{r-2 m}{r-3 m}\right) \frac{r}{2} \frac{\partial}{\partial r}\left(\bar{u}^{a} \bar{u}^{b} h_{a b}\right) .
$$

Eqs. (17) and (22) together imply that

$$
\frac{E^{2} r(r-3 m)}{(r-2 m)^{2}}=1-\bar{u}^{a} \bar{u}^{b} h_{a b}-\frac{r}{2} \frac{\partial}{\partial r}\left(\bar{u}^{a} \bar{u}^{b} h_{a b}\right) .
$$

It is important to note that the $O(\mu)$ parts of $E$ and $J$ depend upon the choice of gauge and have no precise physical meaning in terms of the energy or angular momentum of the particle or the system. There is some irony that $d E / d s$ and $d J / d s$ are gauge independent, while $E$ and $J$ are not.

Two interesting quantities for quasi-circular orbits are components of the four velocity $u^{a}$,

$$
\begin{aligned}
\left(u^{t}\right)^{2} & =\left(\frac{d T}{d s}\right)^{2}=\frac{\left(E+\bar{u}^{b} h_{t b}\right)^{2}}{(1-2 m / r)^{2}} \\
& =\frac{r}{r-3 m}\left(1+\bar{u}^{a} \bar{u}^{b} h_{a b}-\frac{r}{2} \bar{u}^{a} \bar{u}^{b} \partial_{r} h_{a b}\right)
\end{aligned}
$$

and

$$
\begin{aligned}
\left(u^{\phi}\right)^{2} & =\left(\frac{d \Phi}{d s}\right)^{2}=\frac{1}{r^{4}}\left(J-\bar{u}^{b} h_{\phi b}\right)^{2} \\
& =\frac{(r-2 m)}{r(r-3 m)}\left[\frac{m\left(1+\bar{u}^{a} \bar{u}^{b} h_{a b}\right)}{r(r-2 m)}-\frac{1}{2} \bar{u}^{a} \bar{u}^{b} \partial_{r} h_{a b}\right] .
\end{aligned}
$$

These follow from Eqs. (23) and (22) along with the equations in Appendix A.

A gauge transformation is an infinitesimal coordinate transformation $x_{\text {new }}^{a}=x_{\text {old }}^{a}+\xi^{a}$, which transforms $h_{a b} \rightarrow$ $h_{a b}+\Delta h_{a b}$ where $\Delta h_{a b}=-2 \nabla_{(a} \xi_{b)}$. For $h_{a b}$ respecting the symmetry of the helical Killing vector, we show in Appendix B that $\Delta\left(\bar{u}^{a} \bar{u}^{b} h_{a b}\right)=0$ at $\mu$. Thus the right hand side of Eq. (24) changes only in two places. The change in $r /(r-3 m)$ resulting from $r \rightarrow r+\xi^{r}$ is

$$
\Delta\left[\frac{r}{r-3 m}\right]=-\frac{3 m}{(r-3 m)^{2}} \xi^{r}
$$

and the change

$$
\Delta\left(\bar{u}^{a} \bar{u}^{b} \partial_{r} h_{a b}\right)=-\frac{6 m}{r^{2}(r-3 m)} \xi^{r}
$$

is from Eq. (B15). These two changes cancel each other at $O(\mu)$ on the right hand side of Eq. (24), which is therefore gauge independent. A similar argument shows that $u^{\phi}$ is also gauge independent.
Further, the ratio of $u^{\phi}$ and $u^{t}$ gives the orbital frequency

$$
\Omega^{2}=\left(\frac{u^{\phi}}{u^{t}}\right)^{2}=\frac{m}{r^{3}}-\frac{r-3 m}{2 r^{2}} \bar{u}^{a} \bar{u}^{b} \partial_{r} h_{a b}
$$

for a quasi-circular orbit. Its gauge independence is inherited from $u^{t}$ and $u^{\phi}$, and may also be demonstrated directly following the same steps as for $u^{t}$.

While $\Omega$ is observable from infinity and is gauge independent, the Schwarzschild radial coordinate of the orbit is neither. A gauge transformation simply changes the the radius of the orbit by $\Delta r=\xi^{r}$.

\section{Physical interpretations of $u^{t}$}

The quantity $u^{t}$ is the ratio of the rates of change of Schwarzschild coordinate time and of proper time along the particle's geodesic in the perturbed and regularized Schwarzschild metric. We have two different physical interpretations which give further meaning to $u^{t}$.

\section{1. $u^{t}$ as a constant of motion}

The perturbed metric $g_{a b}+h_{a b}$ has a helical Killing vector $k^{a}$. With the reasonable gauge choice that $h_{a b}$ respects the helical symmetry as in Eq. (18), it follows that

$$
-k^{a} u_{a}=E-\Omega J
$$

is a constant of motion over a dynamical timescale. For a general orbit

$$
\begin{aligned}
(E-\Omega J) u^{t} & =E u^{t}-J u^{\phi} \\
& =\frac{E\left(E+u^{b} h_{t b}\right)}{1-2 m / r}-\frac{J}{r^{2}}\left(J-u^{b} h_{\phi b}\right) \\
& =1+\dot{R} \bar{u}^{a} h_{a r}+\dot{R}^{2}\left(\frac{r}{r-2 m}+h_{r r}\right)(30)
\end{aligned}
$$

with Eqs. (8) and (15) being used to derive the second and third equalities, respectively. For a quasi-circular orbit, the right hand side of the last line is $1+O\left(\mu^{2}\right)$, and thus

$$
E-\Omega J=1 / u^{t} .
$$

The gauge independence of $u^{t}$ then implies that the constant of the motion $E-\Omega J$ is also gauge independent even while $E$ and $J$ are not.

$$
\text { 2. } u^{t} \text { as a redshift measurement }
$$

In principle $u^{t}$ might be measured by a redshift observation, which we now describe. 
Let a light source be located at the particle where the perturbed metric is suitably regularized. Let $\nu^{a}$ be the tangent vector to an affinely parameterized null geodesic of a photon from this light source. The energy $\mathcal{E}_{\text {em }}$ of the photon, as emitted, is proportional to $u_{\mathrm{em}}^{a} \nu_{a}$ with $u_{\mathrm{em}}^{a}$ the four velocity of the emitter. Thus the ratio of the energies as measured when observed and when emitted is

$$
\frac{\mathcal{E}_{\mathrm{ob}}}{\mathcal{E}_{\mathrm{em}}}=\frac{u_{\mathrm{ob}}^{a} \nu_{a}}{u_{\mathrm{em}}^{a} \nu_{a}}
$$

with $u_{\mathrm{ob}}^{a}$ the four velocity of the observer. With $k^{a}$ being the helical Killing vector field of the perturbed metric, $k^{a} \nu_{a}$ is a constant of motion along the geodesic of the photon. The four velocity of the emitter is the four velocity of the particle, and $u_{\mathrm{em}}^{a} \propto k^{a}$ so that $u_{\mathrm{em}}^{a}=\left.u^{t} k^{a}\right|_{\mathrm{em}}$. Let the photon be observed along the $z$-axis at a large distance away from the black hole so that $u_{\mathrm{ob}}^{a}=u_{\infty}^{t} \delta_{t}^{a}=\delta_{t}^{a}$. We then have

$$
\frac{\mathcal{E}_{\mathrm{ob}}}{\mathcal{E}_{\mathrm{em}}}=\frac{u_{\infty}^{t} \nu_{t}^{\infty}}{u^{t}\left(k^{a} \nu_{a}\right)_{\mathrm{em}}}=\frac{\nu_{t}^{\infty}}{u^{t}\left(k^{a} \nu_{a}\right)^{\infty}} .
$$

Finally, this may be written as

$$
\begin{aligned}
\frac{\mathcal{E}_{\mathrm{ob}}}{\mathcal{E}_{\mathrm{em}}} & =\frac{\nu_{t}^{\infty}}{u^{t}\left(\nu_{t}^{\infty}+\Omega \nu_{\phi}^{\infty}\right)}=\frac{1}{u^{t}}-\frac{\Omega \nu_{\phi}^{\infty}}{u^{t}\left(\nu_{t}^{\infty}+\Omega \nu_{\phi}^{\infty}\right)} \\
& =\frac{1}{u^{t}}
\end{aligned}
$$

because $\nu_{\phi}^{\infty}=0$ at a large distance along the $z$-axis. Thus, the gauge independent $1 / u^{t}$ determines the redshift of a photon, emitted from the particle, when the photon is observed on the $z$-axis at a large distance.

\section{GAUGE INDEPENDENCE OF OUR RESULTS}

In our analyses, we have a gauge independent expression for the orbital frequency in Eq. (28),

$$
\Omega=\sqrt{\frac{m}{r^{3}}}\left[1-\frac{r(r-3 m)}{4 m} \bar{u}^{a} \bar{u}^{b} \partial_{r} h_{a b}\right]
$$

which allows us to define a related gauge independent measure of the separation between $m$ and $\mu$ via

$$
R_{\Omega} \equiv\left(m / \Omega^{2}\right)^{1 / 3} .
$$

In the geodesic limit, $\mu \rightarrow 0$, the separation $R_{\Omega}$ is precisely the Schwarzschild radial coordinate of the orbit.

Our other gauge independent quantity of interest is $u^{t}$, in Eq. (24), which we now choose to write as

$$
u^{t}={ }_{0} u^{t}+{ }_{1} u^{t}
$$

where

$$
{ }_{0} u^{t} \equiv(1-3 m / r)^{-1 / 2}\left(1-\frac{r}{4} \bar{u}^{a} \bar{u}^{b} \partial_{r} h_{a b}\right)
$$

and

$$
{ }_{1} u^{t} \equiv(1-3 m / r)^{-1 / 2} \frac{1}{2} \bar{u}^{a} \bar{u}^{b} h_{a b} .
$$

From the discussions above and in Appendix B, it is clear that each of these parts of $u^{t}$ are individually gauge independent.

For the moment, assume that the analysis is being performed in a special gauge where $\bar{u}^{a} \bar{u}^{b} \partial_{r} h_{a b}=0$; the gauge vector required to put a general $h_{a b}$ into this special gauge is given in Eq. (B15). In this case

$$
\Omega=\sqrt{\frac{m}{r^{3}}}, \quad R_{\Omega}=r
$$

and

$$
{ }_{0} u^{t}=(1-3 m / r)^{-1 / 2} .
$$

Eqs. (40) and (41) are equivalent to their geodesic limits. It follows that, for this particular choice of gauge,

$$
\begin{aligned}
{ }_{0} u^{t} & =\left(1-3 m / R_{\Omega}\right)^{-1 / 2} \\
& =\left[1-3(\Omega m)^{2 / 3}\right]^{-1 / 2}
\end{aligned}
$$

Every bit of this equation is gauge independent. Therefore Eq. (42) must hold for any choice of gauge. An alternative, direct verification of this equation results from the use of Eqs. (35)-(38) to write both sides of Eq. (42) in terms of $r, m, \bar{u}^{a}$ and $h_{a b}$, followed by the expansion of each side through linear order in $h_{a b}$.

From our perspective the relationships between $\Omega, R_{\Omega}$ and ${ }_{0} u^{t}$ are as if the motion were geodesic in $g_{a b}$ and independent of the self-force and should be treated as such.

To see the effect of the self-force on $u^{t}$, we numerically determine $\bar{u}^{a} \bar{u}^{b} h_{a b}$ and, thence, ${ }_{1} u^{t}$ as a function of $R_{\Omega}$ as described in the next section. We have no need to evaluate $\bar{u}^{a} \bar{u}^{b} \partial_{r} h_{a b}$.

Note that if the motion of $\mu$ were being described as "accelerated in the Schwarzschild geometry", then $u^{a}$ would be normalized by $g_{a b}$. Also $u^{t}$ would not be gauge independent when defined in that manner and the gauge independence of $u^{t}$ with our normalization from $g_{a b}+h_{a b}$ would likely be undiscovered.

\section{DETERMINATION AND REGULARIZATION OF $h_{a b}$}

The major results or our numerical analysis were displayed in Figs. 1 and 2 of Sec. I C. In this section we fill in some details regarding how these results were obtained.

\section{A. The retarded field}

We numerically determine the retarded metric perturbation $h_{a b}^{\text {ret }}$ in the vicinity of a small mass $\mu$ in a circular 


\begin{tabular}{rcclcl}
\hline \hline$R_{\Omega} / m$ & ${ }_{0} u^{t}$ & ${ }_{1} u^{t} m / \mu$ & \multicolumn{1}{c}{$-3 \mathrm{PN}$} & $\bar{u}^{a} \bar{u}^{b} h_{a b}^{\mathrm{R}} / \mu$ & $\mu^{-2} d E / d t$ \\
\hline 5 & 1.581138 & -0.4666524 & -0.14665 & -0.5902738 & 0.002792737 \\
6 & 1.414213 & -0.2960275 & -0.050657 & -0.4186461 & 0.0009403393 \\
7 & 1.322875 & -0.2208475 & -0.022596 & -0.3338900 & 0.0004001632 \\
8 & 1.264911 & -0.1777197 & -0.011704 & -0.2809995 & 0.0001961045 \\
9 & 1.224744 & -0.1493606 & -0.0066994 & -0.2439048 & 0.0001059332 \\
10 & 1.195228 & -0.1291222 & -0.0041222 & -0.2160628 & $6.151631 \times 10^{-5}$ \\
12 & 1.154700 & -0.1019355 & -0.0018198 & -0.1765575 & $2.429170 \times 10^{-5}$ \\
14 & 1.128152 & -0.08438195 & -0.00092714 & -0.1495932 & $1.115762 \times 10^{-5}$ \\
16 & 1.109400 & -0.07205505 & -0.00052185 & -0.1298991 & $5.708047 \times 10^{-6}$ \\
18 & 1.095445 & -0.06290189 & -0.00031616 & -0.1148426 & $3.166609 \times 10^{-6}$ \\
20 & 1.084652 & -0.05582771 & -0.00020271 & -0.1029412 & $1.871470 \times 10^{-6}$ \\
25 & 1.066003 & -0.04359984 & $-7.98 \times 10^{-5}$ & -0.08180055 & $6.157919 \times 10^{-7}$ \\
30 & 1.054092 & -0.03577831 & $-3.75 \times 10^{-5}$ & -0.06788457 & $2.486475 \times 10^{-7}$ \\
35 & 1.045825 & -0.03034064 & $-1.99 \times 10^{-5}$ & -0.05802241 & $1.155513 \times 10^{-7}$ \\
40 & 1.039750 & -0.02633967 & $-1.15 \times 10^{-5}$ & -0.05066538 & $5.950154 \times 10^{-8}$ \\
50 & 1.031421 & -0.02084465 & $-4.65 \times 10^{-6}$ & -0.04041928 & $1.962457 \times 10^{-8}$ \\
60 & 1.025978 & -0.01724759 & $-2.22 \times 10^{-6}$ & -0.03362174 & $7.926444 \times 10^{-9}$ \\
70 & 1.022142 & -0.01470964 & $-1.19 \times 10^{-6}$ & -0.02878197 & $3.681881 \times 10^{-9}$ \\
80 & 1.019294 & -0.01282296 & $-6.94 \times 10^{-7}$ & -0.02516046 & $1.894535 \times 10^{-9}$ \\
90 & 1.017095 & -0.01136531 & $-4.32 \times 10^{-7}$ & -0.02234857 & $1.054112 \times 10^{-9}$ \\
100 & 1.015346 & -0.01020528 & $-2.82 \times 10^{-7}$ & -0.02010207 & $6.238203 \times 10^{-10}$ \\
120 & 1.012739 & -0.008475251 & $-1.35 \times 10^{-7}$ & -0.01673728 & $2.515767 \times 10^{-10}$ \\
140 & 1.010889 & -0.007246793 & $-7.30 \times 10^{-8}$ & -0.01433745 & $1.167029 \times 10^{-10}$ \\
160 & 1.009508 & -0.006329388 & $-4.27 \times 10^{-8}$ & -0.01253953 & $5.998161 \times 10^{-11}$ \\
180 & 1.008438 & -0.005618167 & $-2.66 \times 10^{-8}$ & -0.01114230 & $3.334080 \times 10^{-11}$ \\
200 & 1.007585 & -0.005050642 & $-1.74 \times 10^{-8}$ & -0.01002523 & $1.971428 \times 10^{-11}$ \\
250 & 1.006054 & -0.004032327 & $-7.13 \times 10^{-9}$ & -0.008016120 & $6.476438 \times 10^{-12}$ \\
300 & 1.005037 & -0.003355744 & $-3.43 \times 10^{-9}$ & -0.006677846 & $2.607338 \times 10^{-12}$ \\
\hline \hline & & & & & \\
\hline
\end{tabular}

TABLE I: Summary of the gravitational self-force effects ${ }_{1} u^{t} / \mu, \bar{u}^{a} \bar{u}^{b} h_{a b}^{\mathrm{R}}$ and $d E / d t$ for a variety of radii, $R_{\Omega}$. The label $-3 \mathrm{PN}$ denotes the remainder after the post-Newtonian expansion in Eq. (2) has been removed from ${ }_{1} u^{t}$. 
orbit about a large black hole of mass $m$. We use a standard frequency domain formalism that has been in continuous use for nearly forty years. This formalism is based upon a decomposition of the components of $h_{a b}^{\mathrm{ret}}$ in terms of tensor harmonics. And we rely heavily upon the results of Regge and Wheeler [19] and of Zerilli [14, 15]. We use the Regge-Wheeler gauge for $h_{a b}^{\text {ret }}$, although all of our results are independent of the gauge choice.

\section{B. Regularization}

Two different approaches to regularization have been developed for self-force problems involving point particles and gravitational fields and both lead to the same conclusion. Mino, Sasaki and Tanaka [3] follow the DeWittBrehme [2] analysis for electromagnetic fields in curved spacetime and show that the gravitational self-force may be described completely as geodesic motion in the perturbed geometry $g_{a b}+h_{a b}^{\text {tail }}$, where $h_{a b}^{\text {tail }}$ is the part of the retarded metric perturbation which comes from the support of the Green function within the past null cone of the particle. Quinn and Wald [4] invoke a physically appealing "comparison axiom" and arrive at essentially the same conclusion.

A third approach [5] notes that the tail part of the metric perturbation is only a portion of a solution of the perturbed Einstein equations. However a Green function, different from the retarded Green function, determines the singular part of the metric perturbation $h_{a b}^{\mathrm{S}}$ which exerts no force on the particle. The remainder of the metric perturbation $h_{a b}^{\mathrm{R}} \equiv h_{a b}^{\mathrm{ret}}-h_{a b}^{\mathrm{S}}$ is regular at the particle and is a source-free solution of the perturbed Einstein equations. The self-force is subsequently described as geodesic motion in the combined metric $g_{a b}+h_{a b}^{\mathrm{R}}$. At first perturbative order $g_{a b}+h_{a b}^{\mathrm{R}}$ is a vacuum solution of the Einstein equations, which has the desirable implication that a local observer would see the particle move along a geodesic of a vacuum solution of the Einstein equations and, in fact, would only observe the combined field $g_{a b}+h_{a b}^{\mathrm{R}}$ and have no local method for distinguishing $h_{a b}^{\mathrm{R}}$ from $g_{a b}$.

A practical method for applying the regularization formalism was described first by Barack and Ori [7-10], later by Mino, Nakano and Sasaki $[11,12]$ and subsequently extended by others [20-25]. In this procedure the multipole moments of $h_{a b}^{\mathrm{S}}$ and its derivatives are calculated analytically and referred to as regularization parameters. The sum of these moments diverges when evaluated at the particle, but each individual moment is finite. By construction $h_{a b}^{\mathrm{S}}$ precisely matches the singularity structure of the retarded field at the particle. Thus the difference of the retarded moments and the singular moments gives a multipole decomposition of $h_{a b}^{\mathrm{R}}$ which converges when summed over $l$ and $m$. Schematically, this procedure gives

$$
h_{a b}^{\mathrm{R}}=\sum_{l m} h_{a b}^{\mathrm{R}(l m)}=\sum_{l m}\left[h_{a b}^{\mathrm{ret}(l m)}-h_{a b}^{\mathrm{S}(l m)}\right]
$$

for the regular field.

We show in Sec. III that our problem only requires the regularization of $\bar{u}^{a} \bar{u}^{b} h_{a b}$. Following the original prescription of Barack and Ori [7] and extending it as in Ref. [23], we first evaluate

$$
\bar{u}^{a} \bar{u}^{b} h_{a b}^{\mathrm{ret}(l)} \equiv \sum_{m=-l}^{l} \bar{u}^{a} \bar{u}^{b} h_{a b}^{\mathrm{ret}(l m)} .
$$

Then we use the ansatz that

$$
\begin{aligned}
\bar{u}^{a} \bar{u}^{b} h_{a b}^{\mathrm{S}(l)}= & B+\frac{C}{l+1 / 2}-\frac{D}{(2 l-1)(2 l+3)} \\
& +\frac{E_{1}}{(2 l-3)(2 l-1)(2 l+3)(2 l+5)} \\
& +O\left(l^{-6}\right)
\end{aligned}
$$

where $B, C, D, E_{1}, \ldots$ are the regularization parameters. The particular $l$ dependence of the $D$, and $E_{1}, \ldots$ terms is related to the expansion of $(1-\cos \theta)^{n+1 / 2}$ in terms of Legendre polynomials $P_{l}(\cos \theta)$; details are derived and described in Appendix D of Ref. [23].

For our problem it is known analytically that $C=0$ and

$$
B=2 \sqrt{\frac{r-3 m}{r^{2}(r-2 m)}}{ }_{2} F_{1}\left(\frac{1}{2}, \frac{1}{2}, 1, \frac{m}{r-2 m}\right)
$$

where ${ }_{2} F_{1}$ is a hypergeometric function, and $r$ is the Schwarzschild radial coordinate of the circular orbit. Work in progress will describe the derivation of a variety of regularization parameters, including these. This knowledge of $B$ and $C$, but not $D$, implies that

$$
\bar{u}^{a} \bar{u}^{b} h_{a b}^{\mathrm{R}}=\sum_{l=0}^{\infty}\left(\bar{u}^{a} \bar{u}^{b} h_{a b}^{\mathrm{ret}(l)}-\bar{u}^{a} \bar{u}^{b} h_{a b}^{\mathrm{S}(l)}\right)
$$

converges as $1 / l$. To increase the rate of convergence, we augment our knowledge of $B$ and $C$ by numerically determining further regularization parameters [23]: We use the fact that the behavior of $\bar{u}^{a} \bar{u}^{b} h_{a b}^{\mathrm{ret}(l)}$, evaluated at $\mu$, must match $\bar{u}^{a} \bar{u}^{b} h_{a b}^{\mathrm{S}(l)}$ as given in Eq. (45) for large $l$. This allows us to fit the numerical data to determine the additional regularization parameters $D$, and $E_{1}$ to $E_{3}$. This results in the sum converging as $\sim l^{-9}$. We sum up to $l=40$, providing sufficient accuracy for the results as presented.

A second gauge independent quantity is $d E / d t$ as given in Eq. (19). The regularization parameters for this quantity are all zero, no regularization is required, and the sum over $l$ converges faster than any power of $l$.

Table I has a complete set of the interesting, gauge-independent data regarding a circular orbit of a Schwarzschild black hole. Any other gauge independent quantity, known by us, may be derived from these data. The results for ${ }_{1} u^{t}$ with the post-Newtonian expansion are displayed in Figs. 1 and 2 of Sec. I. 
Barack and Sago [26] have recently used a timedomain formalism to calculate the actual gravitational self-force in the Lorenz gauge. Their $F_{r}$ corresponds to our $\frac{1}{2} \mu \partial_{r}\left(\bar{u}^{a} \bar{u}^{b} h_{a b}^{\mathrm{R}}\right)$. However, we worked in the ReggeWheeler gauge and are not able to compare results.

\section{Gauge difficulties with regularization parameters}

If the singular and retarded fields in Eq. (43) are in different gauges then the schematic description of regularization fails.

The singular field is commonly described in the Lorenz gauge ${ }_{\mathrm{z}} h_{a b}^{\mathrm{S}}$, while the retarded field is easily found in the Regge-Wheeler [19] gauge ${ }_{\mathrm{rw}} h_{a b}^{\mathrm{ret}}$, in the context of the Schwarzschild geometry. By gauge choice ${ }_{\mathrm{rw}} h_{t \phi}=0$ for even-parity perturbations, while ${ }_{\mathrm{z}} h_{t \phi}$ is generally not zero and is singular at $\mu$. In this case ${ }_{\mathrm{rw}} h_{t \phi}^{\mathrm{ret}}-{ }_{{ }_{\mathrm{z}}} h_{t \phi}^{\mathrm{S}}$ is necessarily singular, and the "regularization" procedure fails.

We now show that this failure is circumvented when a gauge independent quantity is calculated.

Assume that the gauge vector relating the two gauge choices is known for the retarded field,

$$
2 \nabla_{(a} \xi_{b)}={ }_{\mathrm{rw}} h_{a b}^{\mathrm{ret}}-{ }_{{ }_{\mathrm{z}}} h_{a b}^{\mathrm{ret}},
$$

and is used to change the retarded field into the Lorenz gauge mode by mode,

$$
\begin{aligned}
{ }_{\mathrm{lz}} h_{a b}^{\mathrm{R}(l m)} & ={ }_{\mathrm{rw}} h_{a b}^{\mathrm{ret}(l m)}-2 \nabla_{(a} \xi_{b)}^{(l m)}-{ }_{\mathrm{lz}} h_{a b}^{\mathrm{S}(l m)} \\
& ={ }_{\mathrm{lz}} h_{a b}^{\mathrm{ret}(l m)}-{ }_{{ }_{\mathrm{z}}} h_{a b}^{\mathrm{S}(l m)} .
\end{aligned}
$$

Each component of ${ }_{\mathrm{lz}} h_{a b}^{\mathrm{R}}$ is now regular and the sum over modes converges.

In this paper, we are eager to calculate the gauge independent combination $\bar{u}^{a} \bar{u}^{b} h_{a b}^{\mathrm{R}}$ for quasi-circular orbits which involves the sum over multipole terms such as

$$
\begin{gathered}
\bar{u}^{a} \bar{u}_{{ }_{\mathrm{z}}}^{b} h_{a b}^{\mathrm{R}(l m)}=\bar{u}^{a} \bar{u}_{\mathrm{rw}}^{b} h_{a b}^{\mathrm{ret}(l m)}-2 \bar{u}^{a} \bar{u}^{b} \nabla_{(a} \xi_{b)}^{(l m)} \\
-\bar{u}^{a} \bar{u}^{b}{ }_{\mathrm{lz}} h_{a b}^{\mathrm{S}(l m)} .
\end{gathered}
$$

The demonstration that $\bar{u}^{a} \bar{u}^{b} h_{a b}$ is gauge independent for quasi-circular orbits does not rely on $h_{a b}$ being the retarded, the singular or the regular field, and it clearly shows that $\bar{u}^{a} \bar{u}^{b} \nabla_{(a} \xi_{b)}^{(l m)}=0$ at the particle, for any gauge vector ${ }^{3}$. Thus the $\nabla_{a} \xi_{b}$ term in Eq. (50) is identically zero, and

$$
\bar{u}^{a} \bar{u}^{b} h_{a b}^{\mathrm{R}}=\sum_{l m}\left[\bar{u}^{a} \bar{u}^{b}{ }_{\mathrm{rw}} h_{a b}^{\mathrm{ret}(l m)}-\bar{u}^{a} \bar{u}^{b}{ }_{\mathrm{lz}} h_{a b}^{\mathrm{S}(l m)}\right]
$$

\footnotetext{
3 The helical symmetry need not be respected by $\xi_{a}$, but the entire discussion of the gauge independence is then substantially more complicated.
}

converges to a value that is independent of any gauge choice.

This technique can be used to regularize any gaugeindependent linear combination of components of $h_{a b}$ and its derivatives. In fact, Moncrief [27] noted that the nonzero components of ${ }_{\mathrm{rw}} h_{a b}$ may be described in terms of gauge independent, linear combinations of components of $h_{a b}$ and its derivatives in a generic gauge. Thus, if the regularization parameters for $\mathrm{rw}_{a b}^{\mathrm{S}}$ are known and if $h_{a b}^{\text {ret }}$ is known in an arbitrary gauge, then what might be termed the regularized gravitational self-force in the Regge-Wheeler gauge could be determined in just this manner. And the result would, at least, be mathematically well defined.

\section{CONCLUSION}

In Ref. [28] Barack and Ori state "The meaningful description of the gravitational self-force must include both $F_{\text {self }}^{\alpha}$ and the metric perturbation $h_{\alpha \beta}$." We agree wholeheartedly with this statement. But we go a step further and believe that the value in calculating the gravitational self-force, in any particular gauge, is to apply it to a question whose answer is related to some physical observable. And a physical observable ought to be independent of the gauge choice. In Appendix $\mathrm{C}$ we give an example of how easily gauge confusion appears even in Newtonian physics where the direction of the gravitational self-force contains no useful physical information without additional knowledge about the coordinates and how the self-force is being evaluated.

Similarly, we prefer to describe the effects of the selfforce as geodesic motion in the perturbed and regularized metric $g_{a b}+h_{a b}$. The alternative description "acceleration $a_{\mathrm{SF}}^{a}$ in $g_{a b}$ " depends upon the gauge choice and bears no relationship to any actual acceleration which an observer moving with the particle would measure with a collection of small masses and springs. At this level of approximation $\mu$ is in free-fall in the actual, physical spacetime metric $g_{a b}+h_{a b}$. To give $a_{\mathrm{SF}}^{a}$ in some gauge seems to imply that $a_{\mathrm{SF}}^{a}$ contains a true physical consequenceif that is the case we would prefer a description of that consequence.

The circular orbits of the Schwarzschild metric perhaps provide the simplest framework for any self-force problem. The angular decomposition and elementary discrete frequency spectrum imply that only ordinary differential equations need to be solved in order to determine the metric perturbations. Apparently, few problems can be formulated in a gauge independent way within this simple framework. We would be surprised if another first-order self-force effect in this particular context were found which did not have a solution in terms of the data available in Table I.

A general orbit has none of the natural symmetry of the quasi-circular orbit. Then none of our $\Omega, u^{t}, u^{\phi}, E$ or $J$ can be described in terms of gauge independent quanti- 
ties. In this situation, where understanding the meaning of a question is likely to be as difficult as determining its answer, perhaps the only significant questions concern the waveform.

However, our preference for Gauge invariance is a matter of taste. So it is important to note that the value of $a_{\mathrm{SF}}^{a}$ in the Lorenz gauge for circular orbits is precisely defined and can be determined in a precise way, as ably demonstrated by Barack and Sago [26]. Their time-domain implementation is sharply focused on $a_{\mathrm{SF}}^{a}$ and takes a significant step in the direction of calculating the elusive self-force effect upon waveforms.

There is reason for optimism regarding waveforms. The difficulty of two disparate length scales for the extreme-mass-ratio problem can be avoided by using the analytically known singular field $h_{a b}^{\mathrm{S}}$ to construct a smoothed out source [26, 29-31] for the wave equation of an approximation to the regular field $h_{a b}^{\mathrm{R}}$. The smoothed out source shows no structure with a length scale of $\mu$. The numerically determined $h_{a b}^{\mathrm{R}}$ can be guaranteed to be at least $C^{2}$ at $\mu[13,31]$. In this case $h_{a b}^{\mathrm{R}}$ directly gives the self-force effects on the motion and simultaneously provides the waveform in the wave zone.

To take full advantage of the self-force formalism will require that $h_{a b}^{\mathrm{R}}$ be evaluated at second-order in $\mu$. But, this impediment does not appear to be fundamental. It is likely that techniques based upon the current successes of numerical relativity will be able finally to reveal a better description of the dissipative effects of the self-force on gravitational waveforms.

\section{Acknowledgments}

The author is grateful for discussions with Leor Barack, Luc Blanchet, Eric Poisson, Norichika Sago, Ian Vega and Bernard Whiting.

Development of the ideas in this paper began while the author was at the Aspen Center for Physics during the 2005 summer workshop LISA Data: Analysis, Sources, and Science; we gratefully acknowledge the Center and the workshop organizers for their support and kind hospitality. These ideas were further developed at the eighth, ninth and tenth Annual Capra meetings at the Rutherford Appleton Laboratory Oxford (2005), University of Wisconsin in Milwaukee (2006) and University of Alabama in Huntsville (2007), respectively. And we are grateful to the organizers of these valuable workshops. The author also acknowledges the University of Florida High-Performance Computing Center (URL: http://hpc.ufl.edu) and the Institute for Fundamental Theory (URL: http://www.phys.ufl.edu/ift) for providing computational resources and support that have contributed to the research results reported in this paper. This work was supported in part by the National Science Foundation, grant No. PHY-0555484.

\section{APPENDIX A: CONVENIENT IDENTITIES FOR GEODESICS OF THE PERTURBED SCHWARZSCHILD GEOMETRY}

The following identities are useful for simplifying an equation by transforming terms involving $E$ and $J$ back and forth into terms only involving $\bar{u}^{a}$. These equations are all elementary consequences of Eq. (9):

$$
\begin{gathered}
\bar{u}^{a} \bar{u}^{b} h_{a b}=\frac{r E}{r-2 m} \bar{u}^{b} h_{t b}+\frac{J}{r^{2}} \bar{u}^{b} h_{\phi b}, \\
\frac{\partial \bar{u}^{a}}{\partial r} \bar{u}^{b} h_{a b}=-\frac{2 m E}{(r-2 m)^{2}} \bar{u}^{b} h_{t b}-\frac{2 J}{r^{3}} \bar{u}^{b} h_{\phi b},
\end{gathered}
$$

and

$$
\begin{aligned}
\frac{2}{r} \bar{u}^{a} \bar{u}^{b} h_{a b}+\frac{\partial \bar{u}^{a}}{\partial r} \bar{u}^{b} h_{a b} & =\frac{2(r-3 m)}{(r-2 m)^{2}} E \bar{u}^{b} h_{t b} \\
\frac{2 m}{r(r-2 m)} \bar{u}^{a} \bar{u}^{b} h_{a b} & +\frac{\partial \bar{u}^{a}}{\partial r} \bar{u}^{b} h_{a b} \\
& =-\frac{2(r-3 m)}{r^{3}(r-2 m)} J \bar{u}^{b} h_{\phi b} .
\end{aligned}
$$

\section{APPENDIX B: GAUGE TRANSFORMATIONS}

The change in the metric perturbation under a gauge transformation $x_{\text {new }}^{a}=x_{\text {old }}^{a}+\xi^{a}$ where $\xi^{a}$ is considered to be infinitesimal (also known as an infinitesimal coordinate transformation) is

$$
\begin{aligned}
\Delta h_{a b}=-\mathcal{L}_{\xi} g_{a b} & =-\xi^{c} \partial_{c} g_{a b}-g_{a c} \partial_{b} \xi^{c}-g_{c b} \partial_{a} \xi^{c} \\
& =\nabla_{a} \xi_{b}-\nabla_{b} \xi_{a} .
\end{aligned}
$$

To preserve reflection symmetry we assume that $\xi^{\theta}$ and its derivatives are all zero on the equatorial plane. Then, for the Schwarzschild geometry we obtain

$$
\begin{gathered}
\Delta h_{r r}=\frac{2 m}{(r-2 m)^{2}} \xi^{r}-\frac{2}{(1-2 m / r)} \partial_{r} \xi^{r}, \\
\Delta h_{t r}=-\partial_{r} \xi_{t}+\frac{2 m}{r(r-2 m)} \xi_{t}-\frac{1}{1-2 m / r} \partial_{t} \xi^{r}, \\
\Delta h_{t t}=\frac{2 m}{r^{2}} \xi^{r}-2 \partial_{t} \xi_{t}, \\
\Delta h_{\phi \phi}=-2 r \xi^{r}-2 \partial_{\phi} \xi_{\phi}, \\
\Delta h_{t \phi}=-\partial_{t} \xi_{\phi}-\partial_{\phi} \xi_{t},
\end{gathered}
$$

and

$$
\Delta h_{r \phi}=-\partial_{r} \xi_{\phi}+\frac{2}{r} \xi_{\phi}-\frac{1}{1-2 m / r} \partial_{\phi} \xi^{r}
$$


For quasi-circular orbits of perturbed Schwarzschild we consider $h_{t t}+\Omega h_{\phi t}$ and $h_{t \phi}+\Omega h_{\phi \phi}$; these are proportional to $\bar{u}^{a} h_{a t}$ and $\bar{u}^{a} h_{a \phi}$ respectively. Under a gauge transformation

$$
\begin{aligned}
\Delta h_{t t}+\Omega \Delta h_{\phi t} & =\frac{2 m}{r^{2}} \xi^{r}-2 \partial_{t} \xi_{t}-\Omega \partial_{t} \xi_{\phi}-\Omega \partial_{\phi} \xi_{t} \\
\Delta h_{t \phi}+\Omega \Delta h_{\phi \phi} & =-\partial_{t} \xi_{\phi}-\partial_{\phi} \xi_{t}-2 \Omega r \xi^{r}-2 \Omega \partial_{\phi} \xi_{\phi}
\end{aligned}
$$

Combining these first provides

$$
\begin{aligned}
\Delta\left(h_{t t}+\right. & \left.2 \Omega h_{t \phi}+\Omega^{2} h_{\phi \phi}\right)=2 r\left(\frac{m}{r^{3}}-\Omega^{2}\right) \xi^{r} \\
& -2\left(\partial_{t}+\Omega \partial_{\phi}\right) \xi_{t}-2 \Omega\left(\partial_{t}+\Omega \partial_{\phi}\right) \xi_{\phi}
\end{aligned}
$$

and subsequently

$$
\begin{aligned}
\bar{u}^{a} \bar{u}^{b} \Delta h_{a b}= & \frac{E^{2}}{(1-2 m / r)^{2}} \Delta\left(h_{t t}+2 \Omega h_{t \phi}+\Omega^{2} h_{\phi \phi}\right) \\
= & \frac{E^{2}}{(1-2 m / r)^{2}}\left[2 r\left(\frac{m}{r^{3}}-\Omega^{2}\right) \xi^{r}\right. \\
& \left.-2\left(\partial_{t}+\Omega \partial_{\phi}\right) \xi_{t}-2 \Omega\left(\partial_{t}+\Omega \partial_{\phi}\right) \xi_{\phi}\right]
\end{aligned}
$$

With the assumption that $\xi^{a}$ respects the helical symmetry $\mathcal{L}_{k} \xi^{a}=\left(\partial_{t}+\Omega \partial_{\phi}\right) \xi^{a}=0$, the above effect of a general gauge transformation simplifies to

$$
\begin{aligned}
\bar{u}^{a} \bar{u}^{b} \Delta h_{a b} & =\frac{E^{2}}{(1-2 m / r)^{2}}\left[2 r\left(\frac{m}{r^{3}}-\Omega^{2}\right) \xi^{r}\right] \\
& =0
\end{aligned}
$$

when evaluated at the particle where $\Omega^{2}=m / r^{3}+O(h)$. Equation (B12) and $\Delta \bar{u}^{a}=O(h)$ then imply that $\Delta\left(\bar{u}^{a} \bar{u}^{b} h_{a b}\right)=0$, at $O(h)$. The fact that

$$
\Delta\left(\bar{u}^{a} \bar{u}^{b} \partial_{t} h_{a b}\right)=0
$$

at the particle, follows simply from Eq. (B11). A similar argument reveals the gauge independence of $\bar{u}^{a} \bar{u}^{b} \partial_{\phi} h_{a b}$ when evaluated at the particle.

It also follows from Eq. (B11) that

$$
\bar{u}^{a} \bar{u}^{b} \frac{\partial}{\partial r} \Delta h_{a b}=\frac{E^{2}}{(1-2 m / r)^{2}}\left(\frac{-6 m}{r^{3}}\right) \xi^{r}
$$

evaluated at the particle. For a quasi-circular orbit $E^{2} /(1-2 m / r)^{2}=1 /(1-3 m / r)+O(\mu)$, so that a general gauge transformation induces a change at the particle

$$
\Delta\left(\bar{u}^{a} \bar{u}^{b} \partial_{r} h_{a b}\right)=-\frac{6 m}{r^{2}(r-3 m)} \xi^{r} .
$$

This result shows that the expression for $\Omega^{2}$ in Eq. (28) is invariant under a general gauge transformation, while the radius of the orbit changes with the radial coordinate, $r_{\text {new }}=r_{\text {old }}+\xi^{r}$, and is not gauge independent.

\section{APPENDIX C: GAUGE DEPENDENCE OF THE NEWTONIAN GRAVITATIONAL SELF-FORCE}

In Newtonian gravity, when one mass $m_{1}$ of a circular binary is infinitesimal, then the orbital frequency is $\Omega^{2}=$ $G m_{2} / r^{3}$. If $m_{1}$ is small but finite, then $\Omega$ changes by $O\left(m_{1} / m_{2}\right)$. This change in $\Omega$ is properly described as a consequence of a Newtonian gravitational self-force [32].

The orbital acceleration of $m_{1}$ in a binary is given by

$$
r_{1} \Omega^{2}=\frac{G m_{2}}{\left(r_{1}+r_{2}\right)^{2}}
$$

where $r_{1}$ and $r_{2}$ are the radii of the orbits and are also the distances from the masses to the center of mass. Thus $m_{1} r_{1}=m_{2} r_{2}$.

Note that

$$
\lim _{m_{1} \rightarrow 0} r_{1}+r_{2}=r_{1}
$$

And in this same limit,

$$
\lim _{m_{1} \rightarrow 0} r_{1} \Omega^{2}=G m_{2} / r_{1}^{2} .
$$

But how does the acceleration of $m_{1}$ change as the limit is taken? The answer would determine the sign of the self-force. For an extreme mass ratio, the magnitude of the acceleration of $m_{1}$ is precisely

$$
r_{1} \Omega^{2}=\frac{G m_{2}}{\left(r_{1}+r_{2}\right)^{2}}
$$

on the one hand, but also

$$
r_{1} \Omega^{2}=\frac{G m_{2}}{\left(r_{1}+r_{2}\right)^{2}}=\frac{G m_{2}}{r_{1}^{2}}\left(1-\frac{2 m_{1}}{m_{2}}+\ldots\right),
$$

on the other.

The acceleration and the self-force depend upon the detail of how $r_{1}+r_{2}$ approaches $r_{1}$. If the distance $r_{1}+$ $r_{2}$ between the masses is held fixed during the limit $\left(r_{1}\right.$ grows slightly while $r_{2}$ shrinks) then Eq. (C4) implies that the acceleration of $m_{1}$ is constant and that there is no self-force. However, if $m_{1}$ 's orbital radius $r_{1}$ is held fixed during the limit, then Eq. (C5) implies that the limit of the acceleration is approached from below, and the self-force points outward.

Even in Newtonian physics the gravitational self-force is ambiguously defined and not particularly relevant to understanding Eq. (C1).

This Newtonian ambiguity is precisely equivalent to the gauge ambiguity of the gravitational self-force in general relativity. For an extreme-mass-ratio binary, if the origin of the Schwarzschild coordinates is at the center of the black hole then the coordinate value of $r$ at the orbit would represent the distance between the particle center and the black hole center, i.e. $r_{1}+r_{2}$, and Eq. (C4) would imply that there is no self-force. However, a dipole gauge transformation can move the origin of the 
coordinates to the center of mass of the system and then the coordinate value of $r$ at the orbit would represent the distance between the particle and the center of mass, i.e. $r_{1}$ alone, and Eq. (C5) would imply that the self-force points outward. Consequently, a simple dipole gauge transformation substantially changes the appearance of the gravitational self-force.

The resolution of this confusion in Newtonian physics is to refrain from introducing the concept of the self-force. It seems reasonable that general relativity would follow the Newtonian lead.

\section{APPENDIX D: USING THE ENERGY FLUX FOR RADIATION REACTION}

For the special case of quasi-circular inspiral, it has long been common knowledge that the dissipative effects of radiation reaction could be calculated by determining ( $i$ ) the total energy of particle $\mu E$, (ii) the orbital frequency $\Omega$, and (iii) the rate of energy loss $d E_{\text {tot }} / d t$ via gravitational waves. Each of these is to be calculated as a function of the radius $r$ of the orbit of $\mu$. As energy is lost $\mu$ slowly spirals inward, and the rate of change of $\Omega$ is then expected to be

$$
\frac{d \Omega}{d t}=\frac{d E_{\mathrm{tot}}}{d t} \times \frac{d \Omega / d r}{\mu d E / d r} .
$$

In this Appendix we show that $d \Omega / d t$ in this equation is equivalent to $d \Omega / d t$ as determined in Eq. (1), which is based upon the self-force formalism.

Equation (D1) and Eq. (1) appear similar but have an important difference. In Eq. (D1), $d E_{\text {tot }} / d t$ is the rate that energy is radiated out at a large distance and down into the black hole, while in Eq. (1) $\mu d E / d t$ is determined locally from $h_{a b}$ via Eq. (19) and is the rate at which the the self-force removes energy from the particle. These two equations give the same $d \Omega / d t$ only if the rate that energy is lost through gravitational waves is equal to the rate that the self-force removes energy from the orbit. We now show that this is the case.

We assume that $g_{a b}$ is the Schwarzschild metric, and only in this appendix an overdot represents a derivative with respect to the Schwarzschild time coordinate $t$.

The perturbed Einstein equations, with a perturbing stress-energy tensor $T_{a b}=O(\mu)$ being the source, may be written as (see Eq. (35.58) of Ref. [33])

$$
16 \pi T_{a b}=-E_{a b}(h)
$$

where

$$
\begin{aligned}
E_{a b}(h)= & \nabla^{2} h_{a b}+\nabla_{a} \nabla_{b} h-2 \nabla_{(a} \nabla^{c} h_{b) c} \\
& +2 R_{a}{ }^{c}{ }_{b}{ }^{d} h_{c d}+g_{a b}\left(\nabla^{c} \nabla^{d} h_{c d}-\nabla^{2} h\right),(\mathrm{D} 3)
\end{aligned}
$$

with $h \equiv h_{a b} g^{a b}$.

For arbitrary symmetric tensors $k^{a b}$ and $h_{a b}$, the operator $E_{a b}(h)$ satisfies the identity [13]

$$
k^{a b} E_{a b}(h)=\nabla_{c} F^{c}(k, h)-\left\langle k^{a b}, h_{a b}\right\rangle
$$

with

$$
F^{c}(k, h) \equiv \bar{k}^{a b} \nabla^{c} \bar{h}_{a b}-\frac{1}{2} \bar{k} \nabla^{c} \bar{h}-2 \bar{k}^{c b} \nabla^{a} \bar{h}_{a b}
$$

where $\bar{h}_{a b} \equiv h_{a b}-\frac{1}{2} g_{a b} h_{c}^{c}$, and similarly for $\bar{k}_{a b}$. Also

$$
\begin{aligned}
\left\langle k^{a b}, h_{a b}\right\rangle \equiv & \nabla^{c} \bar{k}^{a b} \nabla_{c} \bar{h}_{a b}-\frac{1}{2} \nabla^{c} \bar{k} \nabla_{c} \bar{h} \\
& -2 \nabla_{a} \bar{k}^{a c} \nabla^{b} \bar{h}_{b c}-2 \bar{k}^{a b} R_{a b}{ }^{c}{ }^{d} \bar{h}_{c d}
\end{aligned}
$$

is symmetric under interchange of $h_{a b}$ and $k_{a b}$.

Let $k_{a b}=\dot{h}_{a b}$. Then a consequence of Eq. (D2) is

$$
16 \pi T^{a b} \dot{h}_{a b}=-\nabla_{c} F^{c}(\dot{h}, h)+\left\langle\dot{h}^{a b}, h_{a b}\right\rangle
$$

We now evaluate an integral of both sides of this equation over a constant $t$ surface $\Sigma$.

The stress-energy tensor for a point mass moving along a path through space $X^{i}(t)$ is

$$
T^{a b}=\mu \frac{u^{a} u^{b}}{u^{t} \sqrt{-g}} \delta^{3}\left(x^{i}-X^{i}(t)\right) .
$$

So, an integral of the left hand side of Eq. (D7) is

$$
16 \pi \int_{\Sigma} T^{a b} \dot{h}_{a b} r^{2} \sin \theta d r d \theta d \phi=\frac{16 \pi \mu}{u^{t}} u^{a} u^{b} \dot{h}_{a b},
$$

where the right hand side is evaluated at the particle.

Assume that $\Sigma$ is bounded by one two-sphere $\partial \Sigma_{\infty}$ at a large radius and by a second two-sphere $\partial \Sigma_{2 m}$ close to the event horizon. And integrate Eq. (D7) over $\Sigma$. Then substitute Eq. (D9) into the left hand side and the result is

$$
\begin{aligned}
16 \pi \mu \frac{u^{a} u^{b} \dot{h}_{a b}}{u^{t}}= & -\int_{\Sigma} \nabla_{c} F^{c}(\dot{h}, h) r^{2} \sin \theta d r d \theta d \phi \\
& +\frac{1}{2} \frac{d}{d t} \int_{\Sigma}\left\langle h^{a b}, h_{a b}\right\rangle r^{2} \sin \theta d r d \theta d \phi
\end{aligned}
$$

where the time derivative was moved outside the second integral by the virtues of the time independence of the metric and of the symmetry of $\langle$,$\rangle . That same term is$ then zero because $\mu$ is moving along a circular geodesic and $h_{a b}$ respects the helical symmetry $\mathcal{L}_{k} h_{a b}=0$. Thus, the integral is

$$
\begin{array}{r}
16 \pi \mu \frac{u^{a} u^{b} \dot{h}_{a b}}{u^{t}}=-\int_{\Sigma} \partial_{c}\left[r^{2} \sin \theta F^{c}(\dot{h}, h)\right] d r d \theta d \phi \\
=-\frac{d}{d t}\left[\int_{\Sigma} F^{t}(\dot{h}, h) r^{2} \sin \theta d r d \theta d \phi\right] \\
\quad-\int_{\Sigma} \frac{\partial}{\partial x^{i}}\left[r^{2} \sin \theta F^{i}(\dot{h}, h)\right] d r d \theta d \phi \\
=-\left[\oint_{\partial \Sigma_{\infty}}-\oint_{\partial \Sigma_{2 m}}\right]\left[r^{2} \sin \theta F^{r}(\dot{h}, h)\right] d \theta d \phi
\end{array}
$$


where the first term on the right hand side of the second equation is zero because of the helical symmetry, and the third equality follows from Gauss' law.

The definition of $F^{a}(k, h)$ in Eq. (D5) gives

$$
F^{r}(\dot{h}, h)=\dot{\bar{h}}^{a b} \nabla^{r} \bar{h}_{a b}-\frac{1}{2} \dot{\bar{h}} \nabla^{r} \bar{h}-2 \dot{\bar{h}}^{r b} \nabla^{a} \bar{h}_{a b} .
$$

Note that when the integrals in the last line of Eq. (D11) are evaluated close to the event horizon and far into the wave zone $F^{r}(\dot{h}, h)$ is then equal to $32 \pi \mathcal{T}_{t}^{r}$, a component of the effective stress-energy tensor of a gravitational wave in a background geometry as given in Eq. (35.70) of Ref. [33].

Thus, the right hand side of Eq. (D11) is $32 \pi$ times $d E_{\text {tot }} / d t$, the rate that the gravitational waves carry energy out through the boundaries of $\Sigma$. Our conclusion is

$$
\frac{d E_{\mathrm{tot}}}{d t}=-\frac{\mu}{2 u^{t}} u^{a} u^{b} \partial_{t} h_{a b}=\frac{d E}{d t},
$$

so that Eq. (D1) and Eq. (1) have the same implications.

In a numerical study of quasi-circular orbits, a comparison of $d E_{\text {tot }} / d t$ with $\mu d E / d t$ is a useful test of the numerical implementation and accuracy, but is not a test of the self-force formalism.

For completeness, we note that the actual, slow inspiral of a quasi-circular orbit is solely a result of the radiative energy and angular momentum losses which lead to $\mu$ slowly falling inward to a tighter orbit. The radial component of the self-force does no work on $\mu$ and is not responsible for the inspiral.

\section{APPENDIX E: THE POST-NEWTONIAN EXPANSIONS OF $u^{t}$ AND $\Omega$}

Recently we have used the analysis by Blanchet, Faye and Ponsot (BFP) [17, 18] to determine the postNewtonian relationship between the two gauge independent quantities $\Omega$ and $u^{t}$ in the extreme mass ratio limit.

We use many of the results and much of the notation of BFP without extensive clarification, and we limit our interest to orbits which are circular up to the effects of radiation reaction. In this appendix only, $c$ is not set to unity, and an expression of the form $O(n)$ refers to general terms of order $1 / c^{n}$ in the limit $c \rightarrow \infty$.

The two components of the binary system are $m_{1}$ and $m_{2}$, and

$$
M \equiv m_{1}+m_{2}
$$

is the total mass. The components are located at $\vec{y}_{1}$ and $\vec{y}_{2}$ with velocities $\vec{v}_{1}=d \vec{y}_{1} / d t$ and $\vec{v}_{2}=d \vec{y}_{2} / d t$, whose magnitudes $v_{1}$ and $v_{2}$ are given in Eqs. (E15) and (E16) below. For convenience the Cartesian dot product between two vectors is denoted

$$
\left(v_{1} v_{2}\right) \equiv \vec{v}_{1} \cdot \vec{v}_{2}=-v_{1} v_{2}
$$

for the circular orbits of interest.

Also the relative position of the masses is $\vec{r}_{12} \equiv \vec{y}_{1}-\vec{y}_{2}$, and the relative velocity is $\vec{v}_{12} \equiv \vec{v}_{1}-\vec{v}_{2}$. The magnitude of the relative velocity is

$$
v_{12}=\Omega r_{12}+O(6)
$$

where $\Omega$ is the angular velocity. The $O(6)$ term is a consequence of radiation reaction at $2.5 \mathrm{PN}$.

We summarize some results from BFP and Blanchet's Living Review [18]. One dimensionless measure of the strength of the gravitational field is

$$
\gamma \equiv \frac{G M}{r_{12} c^{2}}
$$

and a second is

$$
\frac{v_{12}^{2}}{c^{2}}=\frac{\Omega^{2} r_{12}^{2}}{c^{2}}+O(8) .
$$

These two parameters are precisely equal only in the Newtonian limit,

$$
\gamma=\frac{v_{12}^{2}}{c^{2}}+O(4)
$$

It is natural, then, to introduce a third dimensionless parameter

$$
x^{3} \equiv \gamma^{2} \frac{v_{12}^{2}}{c^{2}}=\gamma^{2} \frac{\Omega^{2} r_{12}^{2}}{c^{2}}=\frac{G^{2} M^{2} \Omega^{2}}{c^{6}}
$$

which has the useful features that $x=v_{12}^{2} / c^{2}+O(4)$ and that it depends only upon the quantities $M$ and $\Omega$ which are independent of any choice of coordinates.

The second dimensionless parameter $v_{12}^{2} / c^{2}$ is known in terms of $\gamma$ at the $2 \mathrm{PN}$ order to be

$$
\frac{v_{12}^{2}}{c^{2}}=\gamma-(3-\nu) \gamma^{2}+\left(6+\frac{41}{4} \nu+\nu^{2}\right) \gamma^{3}+O(8)
$$

as given in BFP Eq. (8.6) or Eq. (190) in Ref. [18], with $\nu=m_{1} m_{2} / M^{2}$. From Eqs. (E7) and (E8)

$$
x^{3}=\gamma^{3}-(3-\nu) \gamma^{4}+\left(6+\frac{41}{4} \nu+\nu^{2}\right) \gamma^{5}+O(12) .
$$

Alternatively, $\gamma$ may be expanded in terms of $x$ which results in

$$
\gamma=x+\left(1-\frac{\nu}{3}\right) x^{2}+\left(1-\frac{65 \nu}{12}\right) x^{3}+O(8)
$$

as in Eq. (193) of ref. [18].

Now we focus on $u^{t}$ and its post-Newtonian expansion via the regularized metric at $m_{1}$ given by BFP in their Eq. (7.6).

Let $V^{a}=\left(c, \vec{v}_{1}\right)$, and let $u^{a}$ be parallel to $V^{a}$ but normalized so that $u^{a} u_{a}=-1$. Thus, $u^{a}=u^{t} V^{a}$ is the appropriately parameterized four velocity of $m_{1}$. In this 
Appendix our major task is to find the post-Newtonian expression for

$$
u^{t}=1 / \sqrt{-V^{a} V^{b} g_{a b}}=1 / \sqrt{\left|g_{t t}+2 v_{1}^{i} g_{i t}+v_{1}^{i} v_{1}^{j} g_{i j}\right|} .
$$

Our restriction to circular orbits allows for simplifications of the more general regularized $2 \mathrm{PN}$ metric at the location of $m_{1}$ given in BFP Eq. (7.6). For example, $\left(n_{12} v_{12}\right) \equiv \vec{r}_{12} \cdot \vec{v}_{12} / r_{12}=O(5)$, and such terms in Eq. (7.6) do not lead to a contribution to $u^{t}$ at $\mathrm{O}(6)$. Starting with BFP Eq. (7.6), specializing to the circular orbit case, and dropping terms whose contributions to $u^{t}$ are smaller than $O(6)$, we obtain the metric components

$$
\begin{aligned}
&\left(g_{00}\right)_{1}=-1+2 \frac{G m_{2}}{c^{2} r_{12}}+\frac{G m_{2}}{c^{4} r_{12}}\left(4 v_{2}^{2}-3 \frac{G m_{1}}{r_{12}}-2 \frac{G m_{2}}{r_{12}}\right)+\frac{G m_{2}}{c^{6} r_{12}}\left(4 v_{2}^{4}\right)+\frac{G^{2} m_{1} m_{2}}{c^{6} r_{12}^{2}}\left(\frac{23}{4} v_{1}^{2}-\frac{39}{2}\left(v_{1} v_{2}\right)\right) \\
&+ \frac{47}{4} \frac{G^{2} m_{1} m_{2}}{c^{6} r_{12}^{2}} v_{2}^{2}+\frac{G m_{2}}{c^{6} r_{12}}\left\{\frac{G m_{2}}{r_{12}}\left[-v_{2}^{2}\right]-\frac{G^{2} m_{1}^{2}}{r_{12}^{2}}+\frac{17}{2} \frac{G^{2} m_{1} m_{2}}{r_{12}^{2}}+2 \frac{G^{2} m_{2}^{2}}{r_{12}^{2}}\right\}+O(8), \\
&\left(g_{0 i}\right)_{1}=-4 \frac{G m_{2}}{c^{3} r_{12}} v_{2}^{i}+\frac{G m_{2}}{c^{5} r_{12}}\left\{4 \frac{G m_{1}}{r_{12}} v_{1}^{i}+v_{2}^{i}\left(-4 v_{2}^{2}-2 \frac{G m_{1}}{r_{12}}+\frac{G m_{2}}{r_{12}}\right)\right\}+O(7), \\
&\left(g_{i j}\right)_{1}=\delta^{i j}+2 \frac{G m_{2}}{c^{2} r_{12}} \delta^{i j}+\frac{G m_{2}}{c^{4} r_{12}} \delta^{i j}\left(\frac{G m_{1}}{r_{12}}+\frac{G m_{2}}{r_{12}}\right)+\frac{G m_{2}}{c^{4} r_{12}}\left\{4 v_{2}^{i} v_{2}^{j}\right\}+O(6) .
\end{aligned}
$$

The order of terms, bracketing and other details in these equations are as close as possible to the original form given in BFP to facilitate a comparison of this form to the original, which an enthusiastic reader might attempt.

In the center of mass frame of reference

$$
v_{1} \equiv\left|\vec{v}_{1}\right|=\left[m_{2}+3 \gamma^{2} \nu\left(m_{1}-m_{2}\right)\right] v_{12} / M+O(6),
$$

and

$$
v_{2} \equiv\left|\vec{v}_{2}\right|=\left[m_{1}-3 \gamma^{2} \nu\left(m_{1}-m_{2}\right)\right] v_{12} / M+O(6),
$$

from Eq. (187) of Ref. [18].

With these substitutions, the use of Eqs. (E12)-(E14) and $G$ being removed in favor of $\gamma$ via Eq. (E4), we obtain

$$
\begin{aligned}
1 / u^{t^{2}}=-V^{a} V^{b} g_{a b}= & 1-\frac{m_{2}{ }^{2} v_{12}^{2}}{M^{2} c^{2}}-2 \frac{m_{2} \gamma}{M}-2 \frac{m_{2}{ }^{3} v_{12}{ }^{2} \gamma}{M^{3} c^{2}}\left(1+4 \mu_{1}+2 \mu_{1}^{2}\right)+\frac{m_{2}{ }^{2} \gamma^{2}}{M^{2}}\left(2+3 \mu_{1}\right)-4 \frac{m_{2}{ }^{3} \mu_{1}^{2} v_{12}^{4} \gamma}{M^{3} c^{4}} \\
& -\frac{m_{2}{ }^{4} v_{12}^{2} \gamma^{2}}{4 M^{4} c^{2}}\left(4+27 \mu_{1}+114 \mu_{1}^{2}+47 \mu_{1}^{3}\right)+\frac{m_{2}{ }^{3} \gamma^{3}}{2 M^{3}}\left(-4-17 \mu_{1}+2 \mu_{1}^{2}\right)+O(8),
\end{aligned}
$$

with $\mu_{1} \equiv m_{1} / M$.

Next $v_{12}^{2} / c^{2}$ is removed in favor of $\gamma$ using Eq. (E8),

$$
\begin{aligned}
1 / u^{t^{2}}= & 1+\left(-3+4 \mu_{1}-\mu_{1}^{2}\right) \gamma+\left(3-10 \mu_{1}+11 \mu_{1}^{2}-5 \mu_{1}^{3}+\mu_{1}^{4}\right) \gamma^{2} \\
& +\left(-3+\frac{1}{2} \mu_{1}+\frac{1}{2} \mu_{1}^{2}-\frac{51}{4} \mu_{1}^{3}-\frac{59}{4} \mu_{1}^{4}+6 \mu_{1}^{5}-\mu_{1}^{6}\right) \gamma^{3}+O(8) .
\end{aligned}
$$

And $\gamma$ is removed in favor of $x$ using Eq. (E10) which yields

$$
\begin{aligned}
u^{t}= & +\left(\frac{3}{2}-2 \mu_{1}+\frac{1}{2} \mu_{1}^{2}\right) x+\left(\frac{27}{8}-\frac{13}{2} \mu_{1}+\frac{53}{12} \mu_{1}^{2}-\frac{4}{3} \mu_{1}^{3}+\frac{1}{24} \mu_{1}^{4}\right) x^{2} \\
& +\left(\frac{135}{16}-\frac{175}{8} \mu_{1}+\frac{409}{16} \mu_{1}^{2}-\frac{97}{6} \mu_{1}^{3}+\frac{69}{16} \mu_{1}^{4}-\frac{1}{4} \mu_{1}^{5}-\frac{1}{48} \mu_{1}^{6}\right) x^{3}+O(8)
\end{aligned}
$$

as the $\mathrm{O}(6)$ expression for $u^{t}$ of $m_{1}$ in a circular binary with $m_{2}$. 
To change from this expansion, appropriate for a comparable mass binary, to a similar one with an extreme mass ratio it is necessary to replace the post-Newtonian dimensionless parameter

$$
x=\left[\frac{\Omega G m_{2}}{c^{3}}\left(1+\frac{m_{1}}{m_{2}}\right)\right]^{2 / 3}
$$

with a dimensionless parameter

$$
x_{\epsilon} \equiv\left(\frac{\Omega G m_{2}}{c^{3}}\right)^{2 / 3}
$$

more convenient for simultaneous, independent expansions in $v / c$ and in $\epsilon \equiv m_{1} / m_{2}$. [Note that $x_{\epsilon}=$ $G m_{2} / R_{\Omega} c^{2}$, from Eq. (36).] It follows that

$$
x=x_{\epsilon}(1+\epsilon)^{2 / 3},
$$

and expanding yields

$$
x=x_{\epsilon}\left[1+\frac{2}{3} \epsilon-\frac{1}{9} \epsilon^{2}+\frac{4}{81} \epsilon^{3}+O\left(\epsilon^{4}\right)\right] .
$$

The final steps are the substitutions of $\mu_{1}=\epsilon /(1+\epsilon)$ and of Eq. (E23) into Eq. (E19) and the subsequent collection of powers of the small quantities $\epsilon$ and of $x_{\epsilon}$. Thus, for an extreme mass ratio with a particle of mass $m_{1}$ in a circular orbit with the orbital frequency $\Omega$ about a
Schwarzschild black hole of mass $m_{2}$

$$
\begin{aligned}
u^{t}= & 1+\left(\frac{3}{2}-\epsilon+\epsilon^{2}-\frac{28}{27} \epsilon^{3}\right) x_{\epsilon} \\
& +\left(\frac{27}{8}-2 \epsilon+3 \epsilon^{2}-\frac{67}{18} \epsilon^{3}\right) x_{\epsilon}^{2} \\
& +\left(\frac{135}{16}-5 \epsilon+\frac{97}{8} \epsilon^{2}-\frac{97}{6} \epsilon^{3}\right) x_{\epsilon}^{3} \\
& +O(8)+O(2) \times O\left(\epsilon^{4}\right) .
\end{aligned}
$$

This result is consistent with the post-Newtonian expansion of the geodesic value of $u^{t}$

$$
\begin{aligned}
{ }_{0} u^{t} & =(1-3 m / r)^{-1 / 2}=\left(1-3 x_{\epsilon}\right)^{-1 / 2} \\
& =1+\frac{3}{2} x_{\epsilon}+\frac{27}{8} x_{\epsilon}^{2}+\frac{135}{16} x_{\epsilon}^{3}+O\left(x_{\epsilon}\right)^{4} .
\end{aligned}
$$

And the first order self-force effect on $u^{t}$ is

$$
{ }_{1} u^{t}=-\epsilon x_{\epsilon}-2 \epsilon x_{\epsilon}^{2}-5 \epsilon x_{\epsilon}^{3}+O\left(\epsilon x_{\epsilon}^{4}\right),
$$

which is plotted in Figs. (1) and (2). In the future, a second-order perturbation analysis might be compared with

$$
{ }_{2} u^{t}=\epsilon^{2} x_{\epsilon}+3 \epsilon^{2} x_{\epsilon}^{2}+\frac{97}{8} \epsilon^{2} x_{\epsilon}^{3}+O\left(\epsilon^{2} x_{\epsilon}^{4}\right) .
$$

[1] S. Drasco, Class. Quant. Grav. 23, S769 (2006).

[2] B. S. DeWitt and R. W. Brehme, Ann. Phys. (N.Y.) 9, 220 (1960).

[3] Y. Mino, M. Sasaki, and T. Tanaka, Phys. Rev. D 55, 3457 (1997).

[4] T. C. Quinn and R. M. Wald, Phys. Rev. D 56, 3381 (1997).

[5] S. Detweiler and B. F. Whiting, Phys. Rev. D 67, 024025 (2003).

[6] E. Poisson, Living Rev. Relativity 7, 6 (2004), http://www.livingreviews.org/lrr-2004-6.

[7] L. Barack and A. Ori, Phys. Rev. D 61, 061502(R) (2000).

[8] L. Barack, Phys. Rev. D 62, 084027 (2000).

[9] L. Barack and A. Ori, Phys. Rev. D 66, 084022 (2002).

[10] L. Barack and A. Ori, Phys. Rev. D 67, 024029 (2003).

[11] Y. Mino, H. Nakano, and M. Sasaki, Prog. Theor. Phys. 108, 1039 (2002).

[12] L. Barack, Y. Mino, H. Nakano, A. Ori, and M. Sasaki, Phys. Rev. Lett. 88, 091101 (2002).

[13] S. Detweiler, Class. Quantum Grav. 22, S681 (2005), http://arxiv.org/abs/gr-qc/0501004.

[14] F. J. Zerilli, Phys. Rev. Lett. 24, 737 (1970).

[15] F. J. Zerilli, Phys. Rev. D 2, 2141 (1970).

[16] E. Poisson, (2004), http://arXiv:gr-qc/0410127.

[17] L. Blanchet, G. Faye, and B. Ponsot, Phys. Rev. D 58,
124002 (1998).

[18] L. Blanchet, Living Reviews in Relativity 9, (2006).

[19] T. Regge and J. A. Wheeler, Phys. Rev. 108, 1063 (1957).

[20] C. O. Lousto, Phys. Rev. Lett. 84, 5251 (2000).

[21] L. M. Burko, Phys. Rev. Lett. 84, 4529 (2000).

[22] L. Barack and C. O. Lousto, Phys. Rev. D 66, 061502(R) (2002).

[23] S. Detweiler, E. Messaritaki, and B. F. Whiting, Phys. Rev. D 67, 104016 (2003).

[24] R. Haas and E. Poisson, Phys. Rev. D 74, 044009 (2006).

[25] R. Haas, Phys. Rev. D 75, 124011 (2007).

[26] L. Barack and N. Sago, Phys. Rev. D 75, 064021 (2007).

[27] V. Moncrief, Ann. Phys. 88, 323 (1974).

[28] L. Barack and A. Ori, Phys. Rev. D 64, 124003 (2001).

[29] L. Barack and D. A. Golbourn, Phys. Rev. D 76, 044020 (2007).

[30] L. Barack, D. A. Golbourn, and N. Sago, Phys. Rev. D 76, 124036 (2007).

[31] I. Vega and S. Detweiler, Phys. Rev. D 77, 084008 (2008).

[32] S. Detweiler and E. Poisson, Phys. Rev. D 69, 084019 (2004).

[33] C. W. Misner, K. S. Thorne, and J. A. Wheeler, Gravitation (Freeman, San Fransisco, 1973). 